(C) 1982. The Genetical Society of Great Britain

\title{
GENETIC LOAD AND SOFT SELECTION IN FERNS
}

\author{
EDWARD J. KLEKOWSKI, JR. \\ Department of Botany, University of Massachusetts, \\ Amherst, Massachusetts 01003, U.S.A.
}

Received 15,iii.82

\section{SUMMARY}

\begin{abstract}
Genetic load data for two homosporous ferns are summarized and the mean frequency of recessive sporophytic lethals per zygote calculated. Fifty-one spore collections of Onoclea sensibilis had a mean of 0.587 lethals per zygote, and 129 spore collections of Osmunda regalis had a mean of 2.39 lethals per zygote. Each spore collection represents a meiotic sample from a single individual (genet). Both species form hermaphroditic gametophytes with extensive capacities for simple polyembryony. Simple polyembryony results in a form of soft selection in panmictic populations and thus higher equilibrium frequencies for lethals are possible than in organisms without simple polyembryony. When ferns are inbred, the high load levels are expressed. Load levels in ferns may reflect a balance between soft selection in panmictic populations and hard selection under conditions of inbreeding. The sheltering effect of simple polyembryony is demonstrated for both mutational and heterotic load.
\end{abstract}

\section{INTRODUCTION}

IN practically all species of sexually reproducing, out-crossing organisms, a low frequency of extreme deviant phenotypes segregates each generation. This observation and the finding that inbreeding such species usually results in a higher frequency of such deviant phenotypes has led to the view that most individuals are heterozygous for deleterious or lethal factors (Dobzhansky, 1957). The concept of genetic load is derived from such observations (Muller, 1950; Morton, Crow and Muller, 1956). Although genetic load has been studied in a diversity of organisms, of special importance to the present research are the studies on Drosophila. In fruit flies special laboratory strains have been developed which segregate to produce progeny homozygous for specific chromosomes; for example, the $C y L / P m$ technique of $D$. melanogaster (Lewontin, 1974). The viabilities of flies completely homozygous for a chromosome are compared to heterozygotes for the same chromosome. In seed plants, such sophisticated techniques for studies of genetic load have been unavailable generally. Genetic loads in these organisms have been estimated by measuring the decline in seed and seedling viability in inbred progeny, (Sorensen, 1969; Franklin, 1972; Bishir and Pepper, 1977) or studying the frequency of heterozygotes for chlorophyll deficiencies (Crumpacker, 1967). Almost a quarter of a century ago, Dobzhansky (1957) noted the paucity of information about genetic load in plants; his observation is still valid for most plants, except the homosporous ferns.

Homosporous ferns have a life cycle consisting of two independent generations, the haploid gametophyte and diploid sporophyte. The meiotic spores produced by many species of these plants can be germinated readily and grown on sterile, defined, agar-solidified medium. These germinated spores grow into hermaphroditic gametophytes, bearing egg-containing 
archegonia and spermatozoid-containing antheridia. The self-fertilization of such gametophytes results in the formation of completely homozygous zygotes because male and female gametes arise through mitotic divisions of the original gametophyte's cells. Thus the gametes are genetically identical (except for mutation) and it follows that syngamy leads to total and complete homozygosity (Klekowski, 1979). Thus recessive deleterious mutations for genes which are either cell lethals or significant in the development of the zygote and embryo will be expressed in these homozygous genotypes. Estimates can be made of the number of genotypes in the population which are heterozygous for deleterious mutations as well as the number of lethals per zygote or per spore. Such data form the basis of estimating the genetic load of a population. Implicit in such studies is the assumption that the fern genotypes contain genes which are restricted in their expression to either the gametophyte or sporophyte generation. The recessive lethals discussed in this communication are restricted to genes expressed only in the sporophyte generation.

\section{Materials AND MEthods}

The methods of fern gametophyte culture and population sampling have been described previously (Klekowski, 1970, 1973). For each spore sample, a gametophyte culture was grown and the frequency of gametophytes capable of developing viable homozygous embryos was determined. The gametophytes which failed to form viable embryos when only selffertilization was possible were classed as bearing recessive sporophytic lethals. The genetic basis of sporophyte inhibition in the latter gametophytes was substantiated by breeding tests (Klekowski, 1970; Schneller, 1979). The frequency of lethal-free meiotic products was used to determine the frequency of lethals per spore and per zygote using the zero term of the Poisson distribution (see Lewontin, 1974 for a discussion of this procedure in Drosophila genetic load studies). In the following studies the distinction between whether a gametophyte genotype carried a sporophytic lethal or was lethal free was based upon the criterion of whether a sporophyte was formed. The future viability of that sporophyte was not considered.

\section{REsults}

(i) Osmunda regalis

$O$. regalis is a member of the fern family, Osmundaceae, whose fossils are found in rocks of Upper Permian age (Taylor, 1981). Genetic load for zygotic lethals was measured for populations from western Massachusetts. Data from Klekowski $(1970,1973)$ as well as studies of populations growing in the nonpolluted parts of the Millers River watershed (see Klekowski and Levin, 1979 for description) were analyzed. The mean number of lethals per zygote was 2.39 and the frequency of lethal-free spores was $0 \cdot 303$. This species has the highest load levels so far documented in the ferns.

$O$. regalis is characterized by a lack of vegetative reproduction in the populations studied; slow growing rhizomes are present which dichotomize 
to form rings 1-1.5 meters in diameter with 8-16 shoot apices. Individual plants may be centuries old. In terms of sexual reproduction, abundant spores are formed in the late spring, but they are green and very short lived ( $48 \mathrm{~h}$ under field conditions). Thus unless suitable environments for germination are encountered within $48 \mathrm{~h}$, the spores lose their viability. Why this species has such a high genetic load is unclear. In this case clonal growth and high genetic load are unrelated and the high load is not compensated for by effective and efficient sexual reproduction.

\section{(ii) Onoclea sensibilis}

Saus and Lloyd (1976) and Ganders (1972) reported the results of genetic load studies for populations from the northeastern part of the United States and Japan. The mean number of lethals per zygote was 0.587 and the frequency of lethal-free spores, $0 \cdot 746 . O$. sensibilis has the capacity for extensive vegetative reproduction. Rapidly growing rhizomes are present which grow at, or close to, the soil surface. Individuals may be large clones composed of hundreds of apices, all of which may form fertile fronds every year. The fertile fronds are woody and remain erect in the winter. Spores are shed in the winter and early spring. Although the spores are green, they survive for many months. This species thus has extensive capacities for clonal and sexual reproduction in contrast to $O$. regalis.

\section{Discussion}

Whether genetic load in ferns is due to mutational load (Muller, 1950; Morton, Crow, and Muller, 1956) or heterotic load (Wright, 1977) is unknown and will not be considered here. The question that will be considered is whether a fern species has considerable genetic load in inbreeding tests, and to what extent does this load hamper sexual reproduction under conditions of random mating and inbreeding? The answer to this question was given 60 years ago by Buchholz (1922) in an analysis of developmental selection and vascular plant life cycles. An important aspect of his idea of developmental selection is the occurrence of simple polyembryony, the formation of a number of different zygotes and embryos on the same gametophyte through the fertilization of multiple archegonia (this is in contrast to cleavage polyembryony which is the vegetative production of more than one embryo from a single zygote).

The gametophytes of many vascular plants (most gymnosperms and pteridophytes) form many archegonia, each containing a single functional egg. Fertilization results in a number of zygotes and later embryos being formed and consequent competition. Since all of the egg genotypes on a single gametophyte are identical, if an egg carries a recessive lethal allele, homozygous and heterozygous genotypes presumably form on the same gametophyte and developmental selection results in heterozygote survival. Thus the selection or loss of the deleterious allele is lessened. The developmental selection of Buchholz is an example of the general concept of soft selection discussed by Wallace (1981). The following will quantify the impact of soft selection based upon simple polyembryony for two models of load, mutational and heterotic. 


\section{(i) Mutational load}

If $p$ is the frequency of the wildtype allele $A$ and $q$ the frequency of the recessive allele $A^{\prime}$, then the array of genotypes after random mating and simple polyembryony is

$$
\begin{array}{lcc}
\text { Genotype } A A & A A^{\prime} & A^{\prime} A^{\prime} \\
\text { Frequency } p^{2} & 2 p q+q^{2} & -
\end{array}
$$

The frequency of $A^{\prime} A^{\prime}$ is zero because gametophytes with $A^{\prime}$ genotypes continued to participate in the breeding pool until a viable embryo $A A^{\prime}$ was formed. The selection coefficient $(s)$ is unity for a recessive lethal and the effective degree of dominance $(h)$ is zero. If $h=0.02$ as has been indicated by Drosophila research (Crow, 1958), then the viability of a zygote heterozygous for a recessive sporophytic lethal is reduced from 1 to 0.98 . If a gametophyte has many archegonia which are fertilized, the probability that at least one forms a viable embryo is $1-h^{n}$ where $n$ is the number of archegonia fertilized. Although the actual number of archegonia fertilized is difficult to measure, Saus and Lloyd (1976) reported that $O$. sensibilis formed on the average 2.87 sporophytes per gametophyte. This is a minimum estimate of simple polyembryony, as the number of zygotes formed by gametophytes is probably greater. Substituting this minimum value, the probability of a gametophyte having a viable heterozygous embryo is $1-0.02^{2 \cdot 87} \cong 1$. Thus because of simple polyembryony and developmental selection, lethals are not selected against in heterozygotes, and in the subsequent equations $h$ can be taken as zero. Therefore average fitness $(\bar{W})$ is unity and consequently genetic load $(L)$ is zero. The frequency of $A^{\prime}$ after one generation is $q_{1}=\left(2 p q+q^{2}\right) / 2 \bar{W}$. The change in allele frequency is $\Delta q=q_{0}-q_{1}$, therefore $\Delta q=q^{2} / 2$. If $u$ is the forward mutation rate of $A \rightarrow A^{\prime}$ and $v$ is the reverse mutation rate $A \leftarrow A^{\prime}$ then the equilibrium allele frequencies can be calculated as follows, $\Delta p=u p-v p$, at equilibrium $\Delta q=\Delta p$; thus, $q^{2} / 2=u(1-q)-v q$. Rewriting this quadratic equation in the standard form,

$$
q^{2}+2 q(u+v)-2 u=0, \quad \hat{q}=\frac{-2(u+v) \pm \sqrt{[2(u+v)]^{2}-4(-2 u)}}{2} .
$$

The equation has only one positive root, thus the equilibrium allele frequency for the lethal allele is $\hat{q} \cong \sqrt{2 u}$, since $u$ is considerably less than 1 and $v$ is one or two orders of magnitude less than $u$. In the absence of simple polyembryony and developmental selection, $\hat{q} \cong u / h s$ and $L=2 u$, where $h>0$ and where $h=0$ and in the absence of simple polyembryony, $\hat{q} \cong \sqrt{u}$ for lethal alleles (Crow and Kimura, 1970).

\section{(ii) Heterotic load}

Simple polyembryony and developmental selection reduce the level of heterotic load in a random mating population to zero also as the following calculations show. The array of genotypes after random mating is

$\begin{array}{ccc}\text { Genotype } A A & A A^{\prime} & A^{\prime} A^{\prime} \\ \text { Fitness }(1-s) & (1) & (1-t) \\ \bar{W}=p^{2}-p^{2} s+2 p q+p^{2} s+q^{2} t+ & q^{2}-q^{2} t\end{array}$


and the average fitness $(\bar{W})$ is unity. As a consequence of $\bar{W}=1$, the genetic load $(L)$ is zero. The allele frequency of $A^{\prime}$ after one generation is

$$
q_{1}=\frac{2 p q+p^{2} s+q^{2} t}{2}+q^{2}-q^{2} t .
$$

The change in allele frequencies is $\Delta q=q_{1}-q_{0}$ and therefore

$$
\Delta q=\frac{p^{2} s-q^{2} t}{2}
$$

The equilibrium allele frequencies may be calculated by making $\Delta q=0$, thus

$$
\begin{gathered}
\frac{p^{2} s}{2}=\frac{q^{2} t}{2}, \\
\hat{q}=\frac{\sqrt{s / t}}{1+\sqrt{s / t}}, \quad \hat{p}=\frac{\sqrt{t / s}}{1+\sqrt{t / s}} .
\end{gathered}
$$

In the absence of soft selection,

$$
\hat{q}=\frac{s}{s+t}, \quad \hat{p}=\frac{t}{s+t}, \quad \text { and } \quad L=\frac{s t}{s+t}
$$

(Crow and Kimura, 1970). Consequently the frequency of heterozygotes in populations is higher where soft selection occurs.

\section{(iii) Inbred load}

In both mutational and heterotic load models, simple polyembryony and developmental selection have three consequences; load levels in randomly mating populations are practically zero, the allele frequencies of lethal and deleterious alleles are higher, and the frequency of heterozygous genotypes is increased. A corollary of such soft selection is a heightened inbreeding depression under conditions where only gametes carrying allelic lethal deleterious mutations are present. In the homosporous ferns such situations often occur in the establishment of new populations. Isolated gametophytes become established which must rely upon their own sperm for self-fertilization. Only homozygous zygotes are formed in spite of repeated fertilizations and simple polyembryony. Since complete homozygosity occurs in one generation of selfing (intragametophytic selfing), there is no alteration in allele frequencies and genetic load can be calculated readily.

In the case of mutational load, inbreeding results in two genotypes with frequencies of $p$ and $q$,

$\begin{array}{ccc}\text { Genotype } & A A & A^{\prime} A^{\prime} \\ \text { Frequency } & p & q \\ \text { Fitness } & 1 & 1-s\end{array}$

average fitness is $\bar{W}=1-s q$, substituting in the equilibrium value for $q$ gives, $\bar{W}=1-s \sqrt{2 u}$. Genetic load expressed under conditions of obligate homozygosity where $s=1$ for lethals is $L=\sqrt{2 u}$. In contrast the mutational 
load expressed under conditions of rapid inbreeding to obligate homozygosity without soft selection is $L=u / h$ where $h>0$ and $L=\sqrt{u}$ where $h=0$ for lethals (Crow and Kimura, 1970).

For the heterotic load model and soft selection, inbreeding to homozygosity results in two genotypes,

$\begin{array}{ccc}\text { Genotype } & \boldsymbol{A A} & \boldsymbol{A} \boldsymbol{A}^{\prime} \\ \text { Frequency } & p & q \\ \text { Fitness } & 1-s & 1-t\end{array}$

The average fitness is, $\bar{W}=1-p s-q t$. The genetic load expressed at total homozygosity is, $L=1-(1-p s-q t) / 1$, substituting the equilibrium allele frequencies,

$$
L=\frac{\sqrt{t / s}}{1+\sqrt{t / s}} s+\frac{\sqrt{s / t}}{1+\sqrt{s / t}} t .
$$

The load for inbreeding to homozygosity without soft selection is $L=$ $2 s t /(s+t)$ for the heterotic load model (Crow and Kimura, 1970).

\section{CONCLUSIONS}

Genetic load levels in fern species vary considerably. Some species have a measurable genetic load of lethal and deleterious mutations whereas other species are essentially free of such mutations (Warne and Lloyd, 1981 ) in inbreeding tests. Although the causes of genetic load in homosporous ferns are not clear (mutational, heterotic, other), given that load occurs, it is possible to show how the life cycle influences the level of load. The majority of homosporous ferns form gametophytes with many archegonia (typically 10 or more). Simple polyembryony and developmental selection reduces the expressed load to zero under conditions of panmixia. The reduced selection against recessive lethals allows their accumulation, and populations are soon characterized by a high frequency of individuals heterozygous for recessive lethals. This leads to an increased expression of genetic load and consequent selection against lethals under conditions of restricted inbreeding, as in the establishment of new populations from single spores. Thus the genetic load levels in a fern species may represent a balance of at least two forces; the build up of load in randomly mating populations in which simple polyembryony counteracts selection against lethal and deleterious homozygotes and heterozygotes, and the loss of load during situations of restricted inbreeding, as in population establishment from single spores. The different load levels in $O$. regalis and $O$. sensibilis may reflect the different ecologies of these species. In New England $O$. regalis is a common but not widespread species; it tends to have more specific environmental requirements in contrast to $O$. sensibilis which is very widespread and is more of a colonizing species. Thus, the high load levels in $O$. regalis are possible because of intergametophytic mating and simple polyembryony, whereas load is reduced in $O$. sensibilis because of more frequent intragametophytic selfing. Such a correlation between load and ecology was documented first by Lloyd (1974) for members of the Hawaiian fern flora. 
Simple polyembryony and developmental selection are not restricted to the homosporous ferns. One would expect this kind of soft selection to occur whenever more than a single archegonium forms on a gametophyte. Among the vascular plants, the majority of extinct and extant gymnosperms and homosporous and heterosporous pteridophytes have been shown to have a multiplicity of archegonia per gametophyte (Taylor, 1981; Bold, 1973).

Acknowledgements. -This paper was written during my tenure as an Alexander von Humboldt Stipendiat at the Albert-Ludwigs-Universität in Freiburg, West Germany. I thank Professor Hans Mohr for his gracious hospitality and stimulating discussions.

\section{REFERENCES}

BISHIR, J., AND PEPPER, W. D. 1977. Estimation of number of embryonic lethal alleles in conifers: I. Self-pollinated seed. Silvae Genetica, 26, 50-54.

BOLD, H. 1973. Morphology of Plants. Harper and Row, New York.

BUCHHOLZ, J. T. 1922. Developmental selection in vascular plants. Bot. Gaz., 73, 249-286. CROW, J. F. 1958. Some possibilities for measuring selection intensities in man. Human Biology, 30, 1-13.

CROW, J. F., AND KIMURA, M. 1970. An Introduction to Population Genetics Theory. Harper and Row, New York.

CRUMPACKER, D. W. 1967. Genetic loads in maize (Zea mays L.) and other cross-fertilized plants and animals. Evolut. Biol., 1, 306-424.

DOBZHANSKY, T. 1957. Genetic loads in natural populations. Science, 126, 191-194.

FRANKLIN, E. C. 1972. Genetic load in loblolly pine. Amer. Natur., 106, 262-265.

GANDERS, F. R. 1972. Heterozygosity for recessive lethals in homosporous fern populations: Thelypteris palustris and Onoclea sensibilis. J. Linn. Soc., Bot., 65, 211-221.

KLEKOWSKI, E. J. JR. 1970. Populational and genetic studies of a homosporous fernOsmunda regalis. Amer. J. Bot., 57, 1122-1138.

KLEKowSKI, E. J., JR. 1973. Genetic load in Osmunda regalis populations. Amer. J. Bot., $60,146-154$

KLEKOWSKI, E. J., JR. 1979. The genetics and reproductive biology of ferns. In The Experimental Biology of Ferns, ed. A. F. Dyer, pp. 133-169. Academic Press, London.

KLEKOWSKI, E. J., JR., AND LEVIN, D. E. 1979. Mutagens in a river heavily polluted with paper recycling wastes: Results of field and laboratory mutagen assays. Environ. Mutagenesis, 1, 209-219.

LEWONTIN, R. C. 1974. The Genetic Basis of Evolutionary Change. Columbia University Press, New York.

LLOYD, R. M. 1974. Mating systems and genetic load in pioneer and non-pioneer Hawaiian pteridophyta. J. Linn. Soc., Bot., 69, 23-35.

MORTON, N. E., CROW, J. F., AND MULLER, H. J. 1956. An estimate of the mutational damage in man from data on consanguineous marriages. Proc. Nat. Acad. Sci., 42, 855-863.

MULLER, H. J. 1950. Our load of mutations. Am J. Human Genet., 2, 111-176.

SAUS, G. L., AND LLOYD, R. M. 1976. Experimental studies on mating systems and genetic load in Onoclea sensibilis L. (Aspleniaceae: Athyrioideae). J. Linn. Soc., Bot., 72, 101-113.

SCHNELLER, J. J. 1979. Biosystematic investigations on the lady fern (Athyrium filix-femina). Pl. Syst. Evol., 132, 255-277.

SORENSEN, F. 1969. Embryonic genetic load in coastal Douglas-fir Pseudotsuga menziesii var. menziesii. Amer. Natur., 103, 389-398.

TAYLOR, T. N. 1981. Paleobotany, An Introduction to Fossil Plant Biology. McGraw-Hill, New York.

WallaCe, B. 1981. Basic Population Genetics. Columbia Press, New York.

WARNE, T. R., AND LLOYD, R. M. 1981. Inbreeding and homozygosity in the fern, Ceratopteris pteridoides (Hooker) Hieronymus (Parkeriaceae). J. Linn. Soc., Bot., 83, 1-13.

WRIGHT, S. 1977. Evolution and the Genetics of Populations, Vol. III. The University of Chicago Press, Chicago. 